Bull. Mater. Sci., Vol. 36, No. 1, February 2013, pp. 135-141. (C) Indian Academy of Sciences.

\title{
Electrical characterization of zirconium substituted barium titanate using complex impedance spectroscopy
}

\author{
PRIYANKA ${ }^{\dagger}$ and A K JHA* \\ Department of Applied Sciences, Ambedkar Institute of Technology (GGSIPU), Geeta Colony, Delhi 110 092, India \\ $\dagger^{\dagger}$ Department of Applied Physics, Delhi Technological University (Formerly Delhi College of Engineering), \\ Bawana Road, Delhi 110 042, India
}

MS received 12 November 2011; revised 10 January 2012

\begin{abstract}
This paper reports complex impedance analysis of polycrystalline complex perovskite structured $\mathrm{BaZr}_{0.025} \mathrm{Ti}_{0.975} \mathrm{O}_{3}$ prepared by solid state reaction method. XRD analysis reveals the formation of single phase perovskite structure. SEM has been used to investigate grain morphology of the material. Impedance plots have been used as a tool to analyse electrical properties of the sample as a function of frequency and temperature. Bulk resistance is observed to decrease with an increase in temperature showing a typical negative temperature coefficient of resistance (NTCR) type behaviour. Nyquist (Cole-Cole) plots show both inter and intra grain boundary effects. Relaxation time is found to decrease with increasing temperature and it obeys the Arrhenius relationship. The variation of d.c. and a.c. conductivity as a function of temperature is also reported.
\end{abstract}

Keywords. Ferroelectrics; barium zirconate titanate; complex impedance spectroscopy.

\section{Introduction}

Complex impedance spectroscopy (CIS) is a nondestructive method (Macdonald 1987) to study the microstructural and electrical properties of polycrystalline solids. This can correlate the structural and electrical characteristics of polycrystalline solids in a wide range of frequencies and temperature. CIS also describes the electrical processes occurring in a system by applying an a.c. signal as input perturbation, which helps to separate the contributions of electro-active regions (such as grain boundary and bulk effects). The impedance plots in the complex plane appear in the form of succession of semicircles representing electrical phenomena due to the bulk material, grain boundary effect and interfacial polarization (Sen et al 2006).

The complex electrical modulus formalism has been used in the analysis of the electrical properties because it gives primary response of the bulk of sample, eliminating the effects due to electrode/electrical contacts. Thus, it is particularly suitable for extracting information due to the electrodes and determining conductivity relaxation times (Hannachi et al 2010). The combined usage of both the impedance and modulus spectroscopy is that $Z^{\prime \prime}$ plots highlight the phenomenon of largest resistance and $M^{\prime \prime}$ plots pick up those of smallest capacitance (Sambasiva Rao et al 2006a).

Barium titanate based ceramics are frequently used in multilayer capacitors, thermistors and other devices owing to their high dielectric constant. The substitution of isovalent and aliovalent cations plays a major role in the modi-

\footnotetext{
*Author for correspondence (prof.akjha@gmail.com)
}

fication of properties of the compound. The substitution of isovalent cation $\mathrm{Zr}^{+4}$ for $\mathrm{Ti}^{+4}$ leads to the formation of barium zirconate titanate (BZT) compound which belongs to the class of complex perovskite structure having the general formula, $\mathrm{AB}_{x} \mathrm{~B}_{1-x}^{\prime} \mathrm{O}_{3}$. These compounds have been widely studied owing to their very high and broad relative permittivity at the ferroelectric Curie point (Clarke and Burfoot 1974; Park 1998). With the substitution of zirconium, permittivity increases considerably due to the interfacial polarization (Costa et al 2011) and interfacial polarization arises due to the discontinuities at the grain boundaries. To understand the conduction mechanism in the zirconium substituted $\mathrm{BaZr}_{0.025} \mathrm{Ti}_{0.975} \mathrm{O}_{3}$, combined effect of impedance and modulus spectroscopy have been studied and reported here.

\section{Experimental}

Polycrystalline samples of composition, $\mathrm{BaZr}_{0.025} \mathrm{Ti}_{0.975} \mathrm{O}_{3}$, were prepared by the solid state reaction method by taking high purity $\mathrm{BaCO}_{3}, \mathrm{TiO}_{2}$ and $\mathrm{ZrO}_{2}$ (all from M/s Aldrich, USA) in their stoichiometric proportions. The powder mixtures were thoroughly ground in an agate mortar and passed through a sieve of appropriate size. The powder mixture was then calcined at $1150^{\circ} \mathrm{C}$ for $2 \mathrm{~h}$ in an alumina crucible. The calcined mixture was ground and admixed with polyvinyl alcohol (PVA) as a binder and then pressed at $150 \mathrm{MPa}$ into disk shaped pellets. The pellets were then sintered at $1300^{\circ} \mathrm{C}$ for $2 \mathrm{~h}$ in air. The sintered pellets (of thickness $0.153 \mathrm{~cm}$ and area $0.865 \mathrm{~cm}^{2}$, measured with the help of screw gauge) were coated with silver paste on both sides to act as electrodes and then finally cured at $300^{\circ} \mathrm{C}$ for $30 \mathrm{~min}$. The dielectric and 
impedance measurements were carried out using a precision LCR meter (HP 4284A) at an oscillation amplitude of $1 \mathrm{~V}$. Impedance measurements were carried out in the temperature range of $300^{\circ} \mathrm{C}$ to $425^{\circ} \mathrm{C}$ at an interval of $25^{\circ} \mathrm{C}$ in the frequency range of $20 \mathrm{~Hz}$ to $1 \mathrm{MHz}$.

\section{Results and discussion}

Before undertaking electrical characterization reported in this paper, the sample was studied for its structural and morphological characteristics, the details of which are reported elsewhere (Jha and Jha 2012).

\subsection{Complex impedance spectrum analysis}

Impedance spectroscopy (IS) is a relatively new and powerful method of characterizing the electrical properties of materials and their interface with electrodes. It may be used to investigate the dynamics of bound or mobile charge in the bulk or interfacial regions of any kind of solid including ionic or mixed electronic-ionic materials and even insulators (Macdonald 1987). The resultant response (when plotted in a complex plane) appears in the form of a succession of semicircles representing contributions due to the bulk, grain boundaries and interfaces. A polycrystalline material usually shows both grain and grain boundary effects with different time constants leading to two successive semicircles (Behera et al 2007a). Electrical properties of a material are often represented in terms of some complex parameters like complex impedance $\left(Z^{*}\right)$, complex admittance $\left(Y^{*}\right)$, complex modulus $\left(M^{*}\right)$, complex permittivity $\left(\varepsilon^{*}\right)$ and dielectric loss (tan $\delta)$. These frequency dependent parameters are related to each other by the following relations (Ganguly et al 2008):

$$
\begin{aligned}
& Z^{*}(\omega)=Z^{\prime}-j Z^{\prime \prime}=R_{\mathrm{s}}-\frac{j}{\omega C_{\mathrm{s}}}=\frac{1}{j \omega C_{\mathrm{o}} \varepsilon^{*}}, \\
& Y^{*}(\omega)=Y^{\prime}+j Y^{\prime \prime}=\frac{1}{R_{\mathrm{p}}}+j \omega C_{\mathrm{p}}=j \omega C_{\mathrm{o}} \varepsilon^{*}=\frac{1}{Z^{*}}, \\
& M^{*}(\omega)=M^{\prime}+j M^{\prime \prime}=\frac{1}{\varepsilon^{*}}=j \omega C_{\mathrm{o}} Z^{*}, \\
& \varepsilon^{*}(\omega)=\varepsilon^{\prime}-j \varepsilon^{\prime \prime}, \\
& \tan \delta=\frac{Z^{\prime}}{Z^{\prime \prime}}=\frac{Y^{\prime}}{Y^{\prime \prime}}=\frac{M^{\prime \prime}}{M^{\prime}}=\frac{\varepsilon^{\prime \prime}}{\varepsilon^{\prime}},
\end{aligned}
$$

where $R_{\mathrm{S}}$ and $R_{\mathrm{p}}$ are the series and parallel resistances, respectively; $C_{\mathrm{s}}$ and $C_{\mathrm{p}}$ the series and parallel capacitances, respectively; $C_{\mathrm{o}}$ the geometrical capacitance; $Z^{\prime}, Y^{\prime}, M^{\prime}, \varepsilon^{\prime}$ and $Z^{\prime \prime}, Y^{\prime \prime}, M^{\prime \prime}, \varepsilon^{\prime \prime}$ denote the real and imaginary components of the impedance, admittance, modulus and permittivity, respectively $(j=\sqrt{-1})$.

Figure 1 shows variation of $Z^{\prime}$ with frequency at different measuring temperatures. A decrease in $Z^{\prime}$ with the increase in frequency and temperature indicates an increase in the a.c. conductivity with an increase in frequency. The coincidence of $Z^{\prime}$ values at higher frequencies at all temperatures indicate a possible release of space charge (Plocharski and Wieczoreck 1988) and a consequent lowering of the barrier properties in the material (Ashok et al 2006).

Figure 2 shows variation of imaginary part of impedance $\left(Z^{\prime \prime}\right)$ with frequency. It provides an insight into the electrical processes having the largest resistance in accordance with relations, $Z^{\prime}=R /\left[1+(\omega R C)^{2}\right]$ and $Z^{\prime \prime}=$ $\omega R^{2} C /\left[1+(\omega R C)^{2}\right]$ (Tiwari and Choudhary 2008). The plots show that $Z^{\prime \prime}$ values attain a peak $\left(Z_{\max }^{\prime \prime}\right)$ at all measuring temperatures. The magnitude of $Z_{\max }^{\prime \prime}$ decreases with temperature indicating decrease in the resistive property of the sample. The value of $Z_{\max }^{\prime \prime}$ shifts to higher frequencies with increasing temperature. This indicates the occurrence of

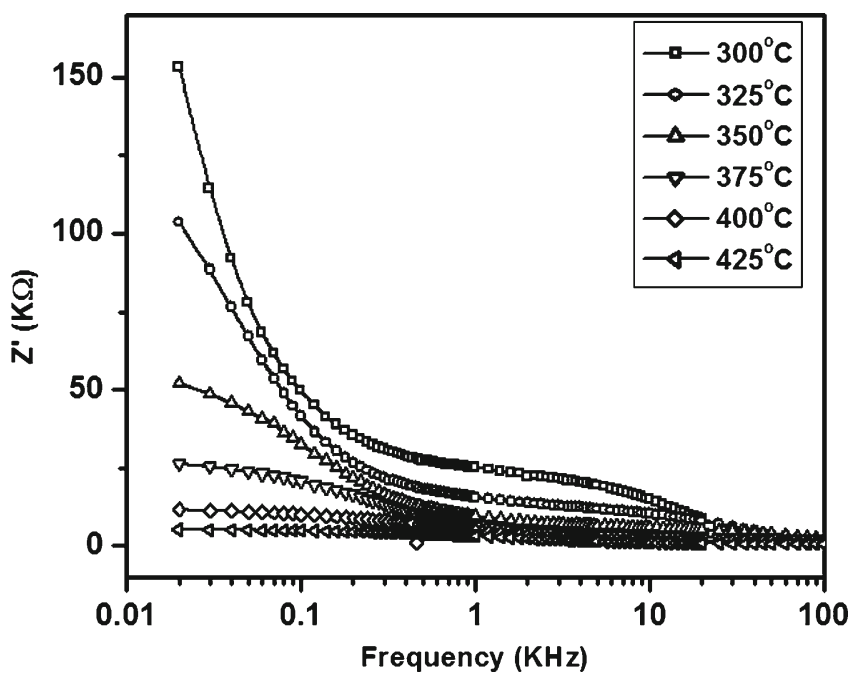

Figure 1. Variation of real part of impedance $\left(Z^{\prime}\right)$ with frequency at different measuring temperatures.

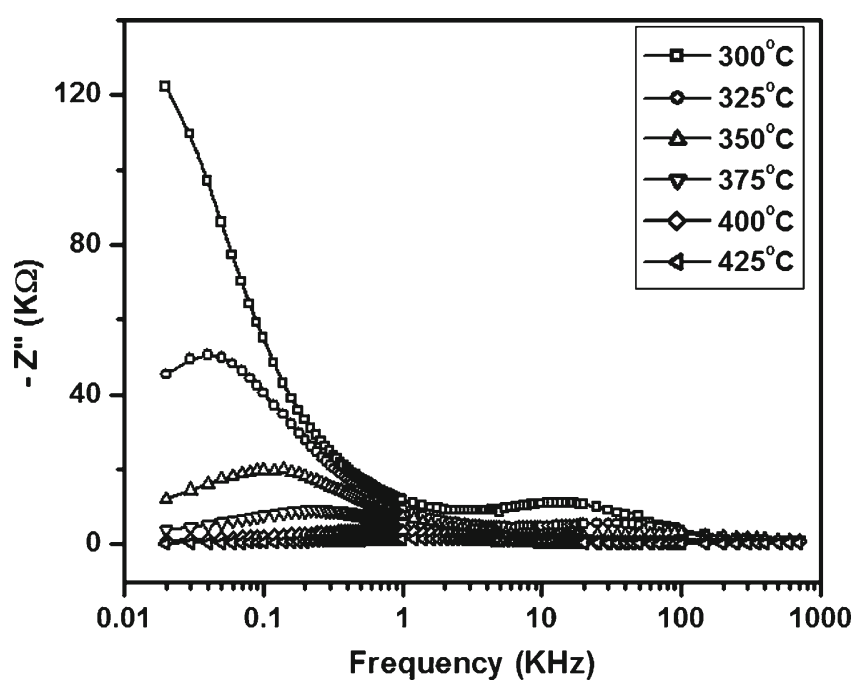

Figure 2. Variation of imaginary part of impedance $\left(Z^{\prime \prime}\right)$ with frequency at different measuring temperatures. 

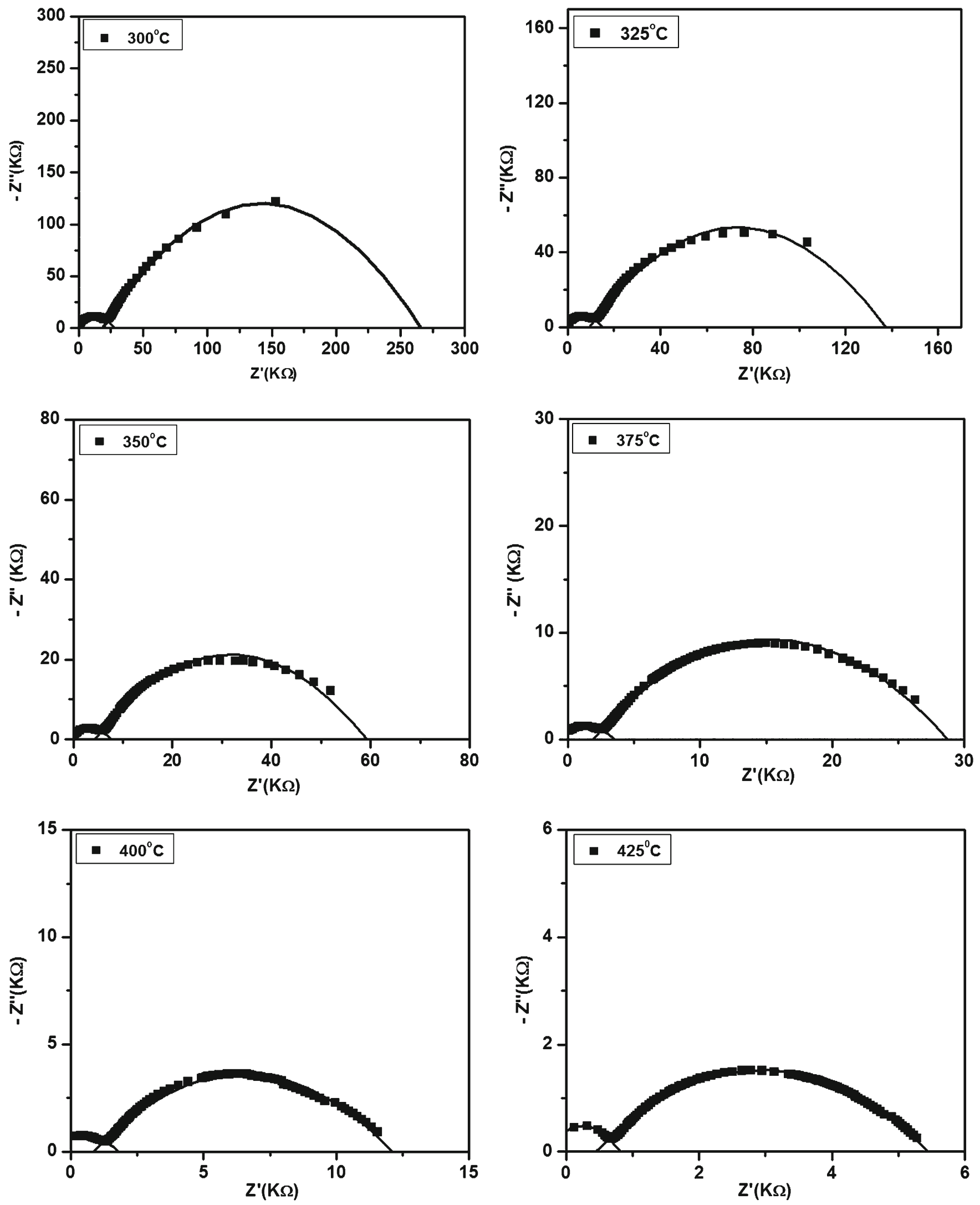

Figure 3. Plot of $\mathrm{Z}^{\prime}$ vs $\mathrm{Z}^{\prime \prime}$ (Nyquist or Cole-Cole plot) taken over a wide frequency range $(20 \mathrm{~Hz}$ to $1 \mathrm{MHz})$ at $300^{\circ} \mathrm{C}, 325^{\circ} \mathrm{C}, 350^{\circ} \mathrm{C}$, $375^{\circ} \mathrm{C}, 400^{\circ} \mathrm{C}$ and $425^{\circ} \mathrm{C}$. 
relaxation in the system (Behera et al 2008). Peak broadening with increase in temperature suggests the presence of temperature dependent electrical relaxation phenomenon in the material (Jonscher 1977). The relaxation process is due to the presence of immobile species at lower temperatures and defects/vacancies at higher temperatures (Tiwari and Choudhary 2008).

Figure 3 shows plot of $Z^{\prime}$ vs $Z^{\prime \prime}$ (Nyquist or Cole-Cole plots) taken over a wide frequency range $(20 \mathrm{~Hz}$ to $1 \mathrm{MHz})$ at different temperatures $\left(300^{\circ} \mathrm{C}\right.$ to $\left.425^{\circ} \mathrm{C}\right)$. This range of temperature and frequency was selected as semicircles are formed in the Nyquist plots within this temperature range. All the semicircles exhibit some depression instead of being centred on the $x$-axis. Such a behaviour is indicative of non- Debye type relaxation and it also manifests that there is a distribution of relaxation time instead of a single relaxation time in the material (Sen et al 2007a). However, presence of two semicircles exhibits the presence of both grain (bulk property) and grain boundary effects. The high frequency semicircle corresponds to a bulk contribution and the low frequency semicircle corresponds to the grain boundary effect (Ganguly et al 2008). The value of bulk resistance $\left(R_{\mathrm{b}}\right)$ and grain boundary resistance $\left(R_{\mathrm{gb}}\right)$ at different temperatures has been obtained from the intercepts of the semicircular arcs on the real axis $\left(Z^{\prime}\right)$. It is observed (also given in table 1) that $R_{\mathrm{b}}$ decreases with increase in temperature. This manifests a negative temperature coefficient of resistance (NTCR) behaviour, a typical characteristic of dielectric materials (Buchanan 1991; Shrivastava et al 2006). The semicircles in the impedance spectrum have a characteristic peak occurring at a unique relaxation frequency $\left(\omega_{\mathrm{r}}=2 \pi f_{\mathrm{r}}\right)$. It can be expressed as: $\omega_{\mathrm{r}} R C=\omega_{\mathrm{r}} \tau=1$. Therefore,

$$
f_{\mathrm{r}}=\frac{1}{2 \pi \tau}=\frac{1}{2 \pi R C},
$$

where $\tau$ is relaxation time. The respective capacitances $\left(C_{\mathrm{b}}\right.$ and $C_{\mathrm{gb}}$ ) due to the grain and grain boundary effects can be calculated using this relation. The values of $R_{\mathrm{b}}, R_{\mathrm{gb}}, C_{\mathrm{b}}$ and $C_{\mathrm{gb}}$ obtained from Cole-Cole plots at different temperatures are listed in table 1.

The relaxation time due to both bulk and grain boundary effects $\left(\tau_{\mathrm{b}}\right.$ and $\tau_{\mathrm{gb}}$ ) has been calculated using (6). Figure 4 shows variation of $\ln \left(\tau_{\mathrm{b}}\right)$ and $\ln \left(\tau_{\mathrm{gb}}\right)$ with the inverse of absolute temperature $(1000 / T)$. It is observed that the value of $\tau_{\mathrm{b}}$ and $\tau_{\mathrm{gb}}$ decreases with an increase in temperature for all the samples and its temperature dependence follows the Arrhenius relation:

$$
\begin{aligned}
\tau_{\mathrm{b}} & =\tau_{\mathrm{o}} \exp \left(\frac{E_{\mathrm{a}}^{\mathrm{b}}}{K_{\mathrm{B}} T}\right), \\
\tau_{\mathrm{gb}} & =\tau_{\mathrm{o}} \exp \left(\frac{E_{\mathrm{a}}^{\mathrm{gb}}}{K_{\mathrm{B}} T}\right),
\end{aligned}
$$

where $\tau_{\mathrm{o}}$ is the pre-exponential factor, $K_{\mathrm{B}}$ the Boltzmann constant and $T$ the absolute temperature. The value of activation energy $\left(E_{\mathrm{a}}\right)$ for the bulk, as calculated from the slope of $\ln \left(\tau_{\mathrm{b}}\right)$ vs $1000 / T$ curve, is observed to be $0.98 \mathrm{eV}$. While the value of activation energy $\left(E_{\mathrm{a}}\right)$ for the grain boundaries, as calculated from the slope of $\ln \left(\tau_{\mathrm{gb}}\right)$ vs $1000 / T$ curve, is observed to be $1.07 \mathrm{eV}$. The conductivity relaxation times are basically dependent on the ion hopping mechanism.

\subsection{Electrical modulus spectrum analysis}

Complex electric modulus formalism/analysis gives information on the nature of polycrystalline (homogeneous or inhomogeneous) samples, which can be resolved into bulk and grain boundary effects. The complex electric modulus

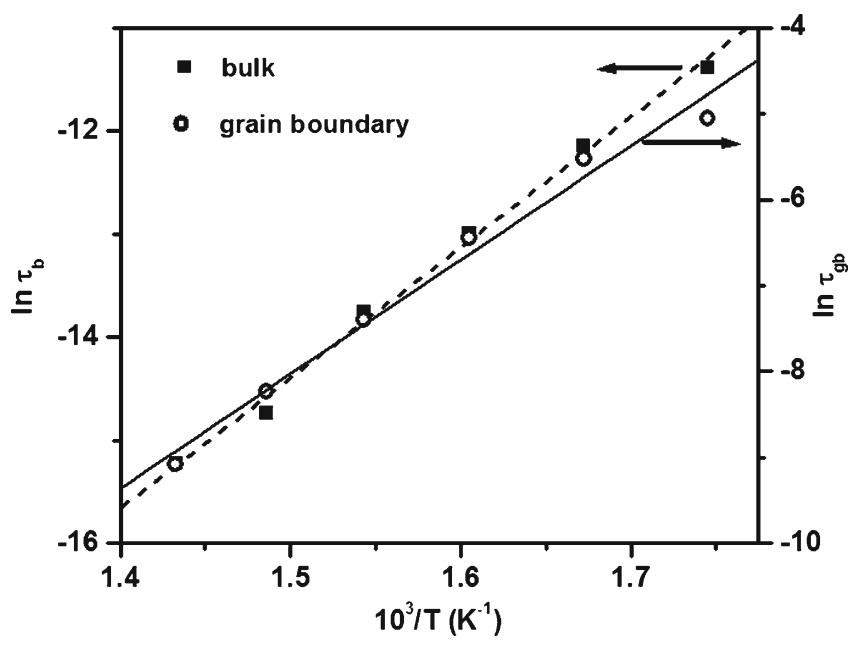

Figure 4. Variation of $\ln \left(\tau_{\mathrm{b}}\right)$ and $\ln \left(\tau_{\mathrm{gb}}\right)$ with inverse of absolute temperature $(1000 / T)$.

\begin{tabular}{|c|c|c|c|c|c|c|}
\hline Temperature $\left({ }^{\circ} \mathrm{C}\right)$ & $R_{\mathrm{b}}(\mathrm{K} \Omega)$ & $R_{\mathrm{gb}}(\mathrm{K} \Omega)$ & $C_{\mathrm{b}}(\mathrm{nF})$ & $C_{\mathrm{gb}}(\mathrm{nF})$ & $\tau_{\mathrm{b}}\left(* 10^{-5}\right)$ & $\tau_{\mathrm{gb}}\left(* 10^{-3}\right)$ \\
\hline 300 & 28.53 & $266 \cdot 62$ & $0 \cdot 40$ & $23 \cdot 89$ & $1 \cdot 14$ & $6 \cdot 37$ \\
\hline 325 & $15 \cdot 59$ & 137.54 & $0 \cdot 34$ & $28 \cdot 94$ & 0.53 & $3 \cdot 98$ \\
\hline 350 & 7.65 & $59 \cdot 39$ & $0 \cdot 30$ & $26 \cdot 81$ & 0.23 & 1.59 \\
\hline 375 & 3.48 & 28.79 & $0 \cdot 31$ & $21 \cdot 27$ & $0 \cdot 10$ & $0 \cdot 61$ \\
\hline 400 & 1.82 & $12 \cdot 14$ & $0 \cdot 22$ & 21.87 & 0.04 & $0 \cdot 26$ \\
\hline 425 & 0.83 & $5 \cdot 46$ & 0.29 & $20 \cdot 82$ & 0.02 & $0 \cdot 11$ \\
\hline
\end{tabular}

Table 1. Bulk resistance $\left(R_{\mathrm{b}}\right)$, bulk capacitance $\left(C_{\mathrm{b}}\right)$, grain-boundary resistance $\left(R_{\mathrm{gb}}\right)$, grain-boundary capacitance $\left(C_{\mathrm{gb}}\right)$, relaxation time for bulk, grain boundary $\left(\tau_{\mathrm{b}}, \tau_{\mathrm{gb}}\right)$ of BZT compound. 
$\left(M^{*}\right)$ was calculated using (3) where $M^{\prime}=\omega C_{\mathrm{o}} Z^{\prime \prime}$ and $M^{\prime \prime}=\omega C_{\mathrm{o}} Z^{\prime}, \quad C_{\mathrm{o}}=\varepsilon_{\mathrm{o}} A / t$ ( $\varepsilon_{\mathrm{o}}$ is permittivity of free space, $A$ the area of the electrode surface and $t$ the thickness of the sample). The complex modulus spectrum ( $M^{\prime}$ vs $M^{\prime \prime}$ ) is shown in figure 5. It indicates electrical phenomenon with the smallest capacitance occurring in a dielectric system (Sen et al 2007b). The appearance of a single arc in the spectrum confirms single phase characteristic of the ceramics. Moreover, the asymmetric semicircular arc confirms the presence of electrical relaxation phenomena in the material (Behera et al 2007b).

Figure 6 shows variation of $M^{\prime}$ with frequency at temperatures mentioned earlier. It is characterized by a very low value (almost zero) of $M^{\prime}$ in the low frequency region followed by a continuous increase with increasing frequency, having a tendency to saturate at a maximum asymptotic value in the high frequency region at all the measuring temperatures. This can be attributed to the presence of conduction phenomena due to short-range mobility of charge carriers (Das et al 2007).

Figure 7 shows frequency dependence of the imaginary part of the electrical modulus $\left(M^{\prime \prime}\right)$. The asymmetric modulus peaks shift towards higher frequency side, indicating correlation between motions of mobile ion charges. The asymmetry in peak broadening shows the spread of relaxation times with different time constants and hence relaxation is of non-Debye type. The existence of low-frequency peaks suggest that the ions can move over long distances, whereas high-frequency peaks suggest confinement of ions in their potential well (Dutta and Sinha 2006). The nature of modulus spectrum confirms the existence of hopping mechanism in the electrical conduction of the material (Costa et al 2011). This is in contrary to an earlier report (Sinclair and West 1989) showing that there are two peaks in the plot of $M^{\prime \prime}$ with frequency in the case of barium titanate attributing the two peaks to two parallel RC combinations. The appearance of only single peak in the present case of zirconium

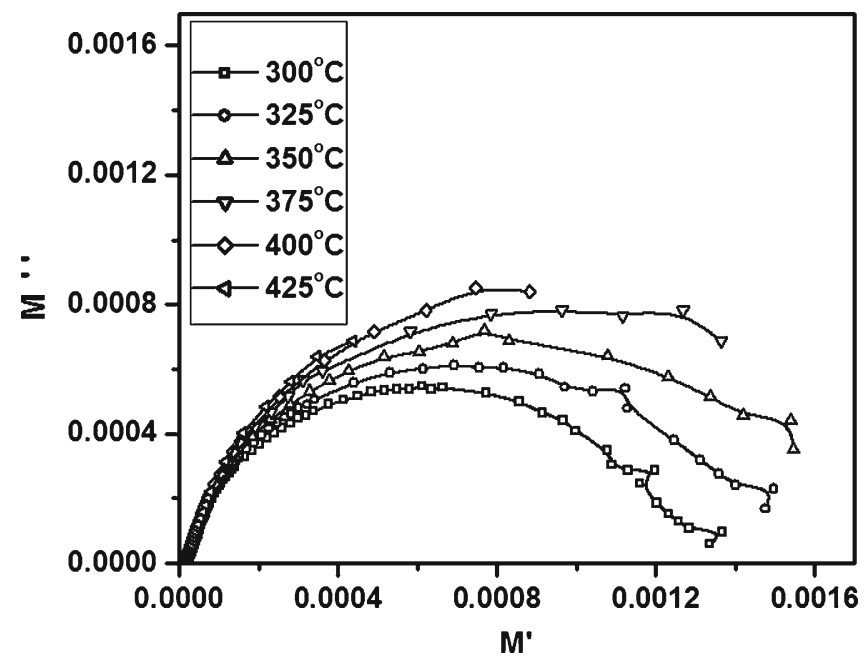

Figure 5. Complex modulus spectrum ( $M^{\prime}$ vs $\left.M^{\prime \prime}\right)$ at different measuring temperatures. substituted barium titanate can be attributed to the fact that the magnitude of the bulk capacitance is considerably lower than that of grain boundary capacitance. Moreover, the $M^{\prime \prime}$ peak frequencies increase as temperature increases. The shift of the frequency of $M^{\prime \prime}$ peaks corresponds to the conductivity relaxation (Sambasiva Rao et al 2006b). The above results are based on the distribution of individual relaxation times (Macdonald 2009).

Scaling behaviour of the sample was studied by plotting normalized parameters (i.e. $\left.M^{\prime \prime} / M_{\max }^{\prime \prime}\right)$ vs $\log \left(f / f_{\max }\right)$, where $f_{\max }$ is the frequency corresponding to $M_{\max }^{\prime \prime}$ at different measuring temperatures (figure 8). The modulus scaling behaviour gives an insight into the dielectric processes occurring inside the material. The low frequency side of the peak in $M^{\prime \prime} / M_{\max }^{\prime \prime}$ vs $\log \left(f / f_{\max }\right)$ curve represents the range of frequencies in which the charge carriers can move over a long distance (i.e. the charge carriers

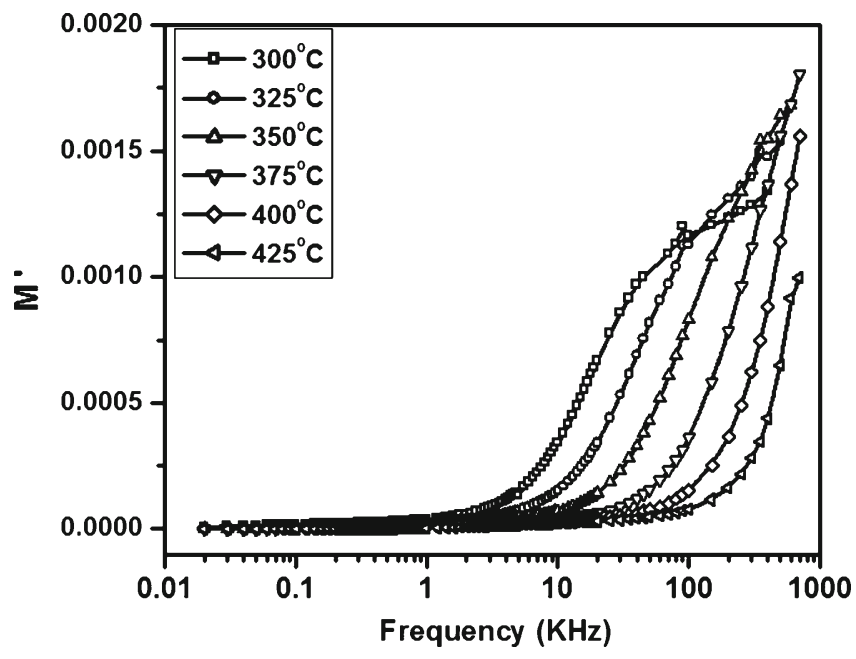

Figure 6. Variation of $M^{\prime}$ with frequency at different temperatures.

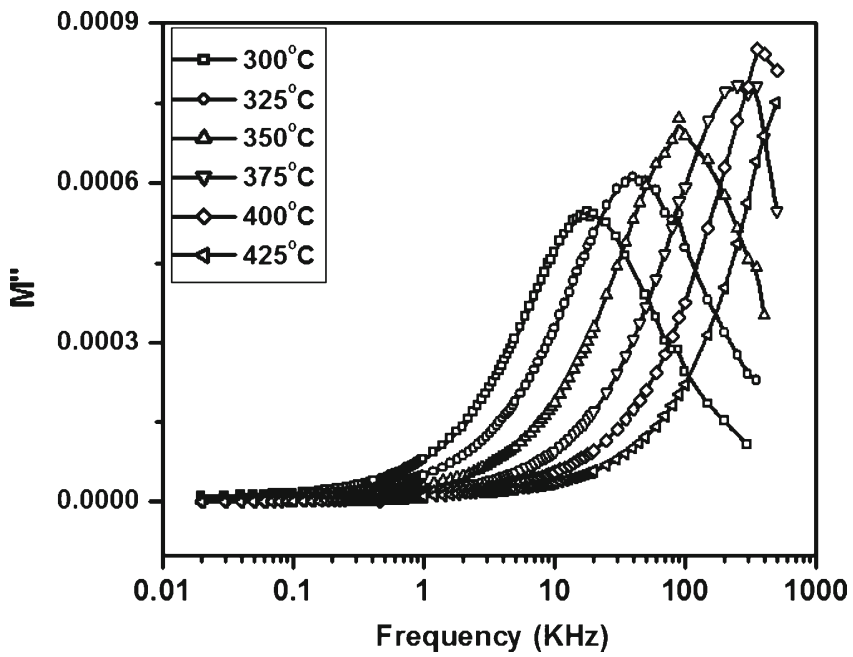

Figure 7. Frequency dependence of imaginary part of electrical modulus $\left(M^{\prime \prime}\right)$. 


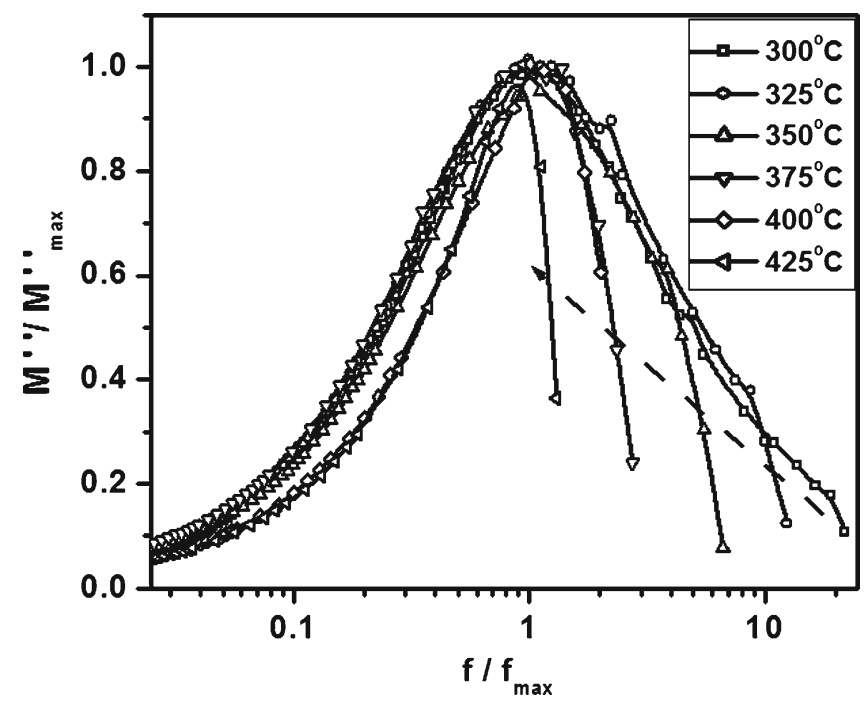

Figure 8. Normalized parameters (i.e. $M^{\prime \prime} / M_{\max }^{\prime \prime}$ ) vs $\log$ $\left(f / f_{\max }\right)$ at different measuring temperatures.

can perform successful hopping from one site to the neighbouring site), whereas the high frequency side of $M^{\prime \prime} / M_{\max }^{\prime \prime}$ vs $\log \left(f / f_{\max }\right)$ curve represents the range of frequencies in which the charge carriers are spatially confined to their potential wells and thus could make localized motions inside the well. The region where the peak occurs is an indication of the transition from long-range to short-range mobility with an increase in frequency (Das et al 2007). The coincidence of all the peaks at different temperatures below the common value of $\log \left(f / f_{\max }\right) \sim 10^{-3}$ indicates temperature dependent behaviour of the relaxation process in the material (Saha and Sinha 2002).

\subsection{Electrical conductivity analysis}

The bulk conductivity $\left(\sigma_{\mathrm{dc}} / \sigma_{\text {bulk }}\right)$ of the material, a thermally activated process obeying Arrhenius behaviour, is estimated in terms of bulk resistance, $R$, evaluated from the impedance spectrum using the relation

$$
\sigma_{\mathrm{dc}}=\frac{t}{R A},
$$

where $R$ is the resistance of the component, $t$ the thickness and $A$ the surface area of the sample.

Figure 9 shows variation of $\sigma_{\mathrm{dc}}$ with $1000 / T$ in case of bulk while the variation of $\sigma_{\mathrm{dc}}$ with $1000 / T$ for the grain boundaries is shown in figure 10. It is observed to increase with increasing temperature further confirming NTCR behaviour. The nature of variation is linear and follows the Arrhenius relationship:

$$
\sigma_{\mathrm{dc}}=\sigma_{\mathrm{o}} \exp \left(\frac{-E_{\mathrm{a}}}{K_{\mathrm{B}} T}\right),
$$

where $E_{\mathrm{a}}$ is the activation energy of conduction and $T$ the absolute temperature. The value of activation energy $\left(E_{\mathrm{a}}\right)$ as calculated from the slope of $\ln \sigma_{\mathrm{dc}}$ vs $10^{3} / T$ curve is found

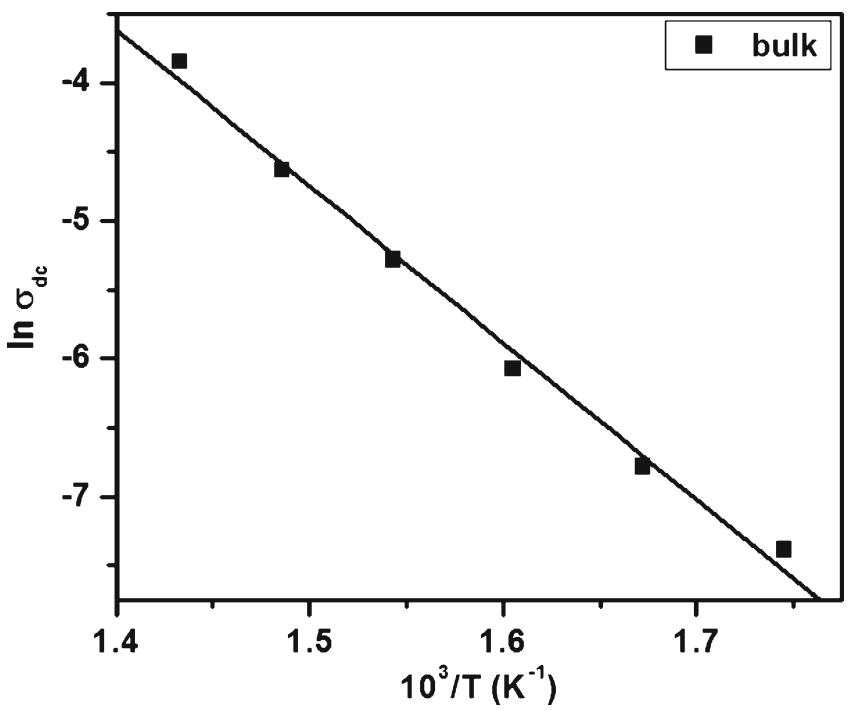

Figure 9. Variation of $\sigma_{\mathrm{dc}}$ with $1000 / T$ in case of bulk.

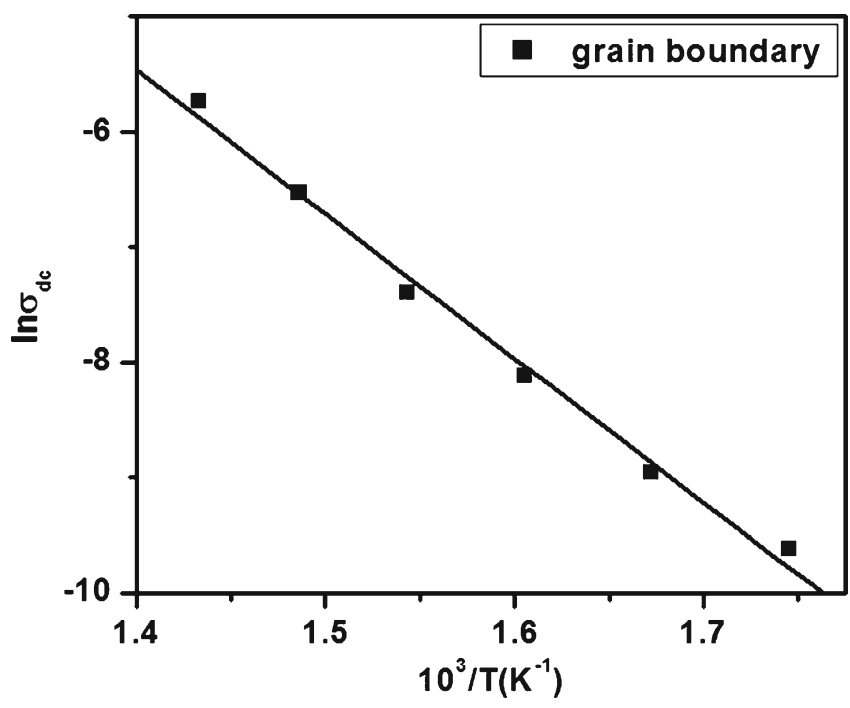

Figure 10. Variation of $\sigma_{\mathrm{dc}}$ with $1000 / T$ for grain boundary.

to be almost the same as that obtained from relaxation time $\left(\tau_{\mathrm{b}}\right)$ vs temperature curve. This value of $E_{\mathrm{a}}$ is approximately the same as the energy required $(\sim 1 \mathrm{eV})$ for the motion of oxygen vacancies (Jonscher 1977). At all the temperatures the conductivity of the grain is higher than that of grain boundaries confirming the high resistive behaviour of grain boundaries.

The a.c. electrical conductivity was obtained using the relation, $\sigma_{\mathrm{ac}}=d / A Z^{\prime}$ (Dutta and Sinha 2007), where $d$ is the thickness and $A$ the surface area of the specimen. The plot of electrical conductivity vs frequency at different measuring temperatures is shown in figure 11. The plot shows that the conductivity increases with increasing temperature. The pattern of conductivity shows some typical features such as (i) dispersion throughout the frequency range of investigation 


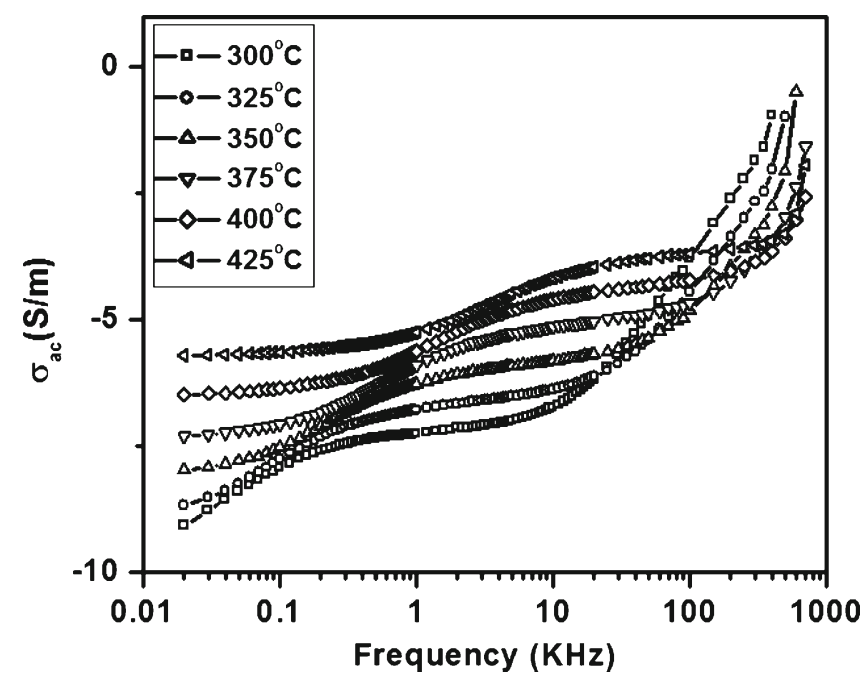

Figure 11. Plot of electrical conductivity vs frequency at different measuring temperatures.

and (ii) low frequency plateau and high frequency dispersion with the change of slope. The frequency at which the change in slope occurs is known as hopping frequency (Srinivas et al 2003). Hopping frequency is observed to shift towards higher frequency side with increase of temperature. In the higher frequency region the increase in conductivity is due to the hopping of charge carrier in finite clusters. The nearly frequency independent region in figure 11 indicates the long range movement of mobile charge carriers (Rout et al 2009).

\section{Conclusions}

The polycrystalline samples of composition, $\mathrm{BaZr}_{0.025} \mathrm{Ti}_{0.975} \mathrm{O}_{3}$, were prepared by the solid state reaction method. X-ray diffraction analysis reveals the formation of single phase perovskite structure. SEM micrograph shows well developed grains with almost no porosity. Nyquist plots show both grain and grain boundary effects. It also shows that bulk resistance $\left(R_{\mathrm{b}}\right)$ decreases with increase in temperature, manifesting the NTCR behaviour of the compound. Relaxation time is found to decrease with increasing temperature and it obeys the Arrhenius relationship. The electrical relaxation process occurring in the material has been found to be temperature dependent. Modulus analysis indicates: (i) presence of single phase, (ii) non-Debye type of conductivity relaxation and (iii) the possibility of hopping mechanism for electrical transport processes.

\section{References}

Ashok Kumar, Singh B P, Choudhary R N P and Thakur Awalendra K 2006 Mater. Chem. Phys. 99150

Behera B, Nayak P and Choudhary R N P 2007a J. Alloys Compds 436226

Behera B, Nayak P and Choudhary R N P 2007b Mater. Chem. Phys. 106193

Behera B, Nayak P and Choudhary R N P 2008 Mater. Res. Bull. 43 401

Buchanan R C 1991 Principles of electronic ceramics (New York: Marcel Dekker) p. 250

Clarke R and Burfoot J C 1974 Ferroelectrics 8505

Costa M M, Pires Jr. G F M, Terezo A J, Grac M P F and Sombra A S B 2011 J. Appl. Phys. 110034107

Das P S, Chakraborty P K, Behera B and Choudhary R N P 2007 Phys. B395 98

Dutta Alo and Sinha T P 2006 J. Phys. Chem. Solids 671484

Dutta Alo and Sinha T P 2007 Phys. Rev. B76 155113

Ganguly Prasun, Jha A K and Deori K L 2008 Solid State Commun. 146472

Hannachi N, Chaabane I, Guidara K, Bulou A and Hiel F 2010 Mater. Sci. Engg. B172 24

Jha Priyanka A and Jha A K 2012 J. Alloys Compds 513580

Jonscher A K 1977 Nature 267673

Macdonald J R 1987 Impedance spectroscopy: Emphasizing solid state material and systems (New York: Wiley) ch. 2 and ch. 4

Macdonald J R 2009 J. Phys. Chem. Solids 70546

Park Y 1998 J. Am. Ceram. Soc. 811957

Plocharski J and Wieczoreck W 1988 Solid State Ionics 28-30 979

Rout S K, Hussain Ali, Lee J S, Kim I W and Woo S I 2009 J. Alloys Compds 477706

Saha S and Sinha T P 2002 Phys. Rev. B65 134103

Sambasiva Rao K, Madhava Prasad D, Murali Krishna P, Tilak B, Varadarajulu K Ch 2006a Mater. Sci. Engg. B133 141

Sambasiva Rao K, Murali Krishna P, Swarnlatha T and Madhava Prasad D 2006b Mater. Sci. Engg. B131 127

Sen Shrabanee, Choudhary R N P, Tarafdar Abhijit and Pramanik P 2006 J. Appl. Phys. 991241141

Sen S, Choudhary R N P and Pramanik P 2007a Physica B387 56

Sen S, Pramanik P and Choudhary R N P 2007b Ceram. Int. 33579

Shrivastava V, Jha A K and Mendiratta R G 2006 Physica B371 337

Sinclair D C and West A R 1989 J. Appl. Phys. 663850

Srinivas K, Sarah P and Suryanarayana S V 2003 Bull. Mater. Sci. 26247

Tiwari Balgovind and Choudhary R N P 2008 J. Phys. Chem. Solids 692852 